\title{
Neonatal Gardner Fibroma: A Sentinel Presentation of Severe Familial Adenomatous Polyposis
}

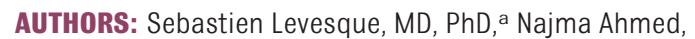
MD, ${ }^{\mathrm{b}}$ Van-Hung Nguyen, MD, ${ }^{\mathrm{c}}$ Ayoub Nahal, MD, ${ }^{\mathrm{c}}$ Miriam Blumenkrantz, MD, ${ }^{\mathrm{c}}$ Pramod Puligandla, MD, MSc, ${ }^{\mathrm{d}}$ George Chong, PhD, e and William D. Foulkes, MBBS, PhDa,f

Departments of a Medical Genetics, ${ }^{b}$ Pediatrics, ${ }^{c}$ Pathology, and aPediatric General Surgery, McGill University Health Centre, Montreal, Quebec, Canada; e Molecular Pathology Unit, Department of Pathology, Jewish General Hospital, Montreal, Quebec, Canada; and Program in Cancer Genetics, Departments of Human Genetics and Oncology, McGill University, Montreal, Quebec, Canada

\section{KEY WORDS}

Gardner syndrome, familial adenomatous polyposis, Gardner fibroma

\section{ABBREVIATIONS}

FAP-familial adenomatous polyposis

GAF-Gardner fibroma

www.pediatrics.org/cgi/doi/10.1542/peds.2010-1045

doi:10.1542/peds.2010-1045

Accepted for publication Jul 31, 2010

Address correspondence to William D. Foulkes, MBBS, PhD, Montreal General Hospital, 1650 Cedar Ave, Montreal, Quebec, Canada H3G 1A4. E-mail: william.foulkes@mcgill.ca

PEDIATRICS (ISSN Numbers: Print, 0031-4005; Online, 1098-4275).

Copyright (? 2010 by the American Academy of Pediatrics

FINANCIAL DISCLOSURE: The authors have indicated they have no financial relationships relevant to this article to disclose.

\section{abstract}

Familial adenomatous polyposis (FAP) is a rare cause of colorectal cancer and rarely presents in early childhood. Various extracolonic manifestations, however, may be present before the development of overt polyposis. One of the rarest manifestations is the Gardner fibroma (GAF), which has particular histologic features. Here we report the case of a child who presented in the neonatal period with a paraspinal mass. Although the initial diagnosis was unclear, biopsy of a second lesion at 32 months of age, and a review of the first lesion, resulted in the diagnosis of GAF. After rectal bleeding at 47 months, colonoscopy revealed 75 to 100 colonic polyps. Adenomas were identified in multiple biopsies throughout the colon and from several polyps located in the duodenum. Polyps were visualized in the jejunum by wireless-capsule endoscopy. A total proctocolectomy was performed, and no malignant transformation was observed in the colon on pathologic inspection. A truncating mutation in APC (c.4479_4480delGG p.Glu1494LysfsX19) was identified in the child. Her parents and sister do not carry this mutation in lymphocyte DNA. To our knowledge, this is the first report of neonatal GAF as the presenting feature of a molecularly confirmed case of sporadic FAP and the earliest colonic and small bowel involvement reported of FAP. It illustrates the need to exclude FAP in a child who harbors fibromas suggestive of GAF, even in the absence of supportive evidence of FAP in the patient or relatives. Pediatrics 2010;126:e00 
Familial adenomatous polyposis (FAP) is a rare cause of colorectal cancer and accounts for fewer than $1 \%$ of cases. ${ }^{1}$ The usual onset of the disease is in the second decade, and hundreds to thousands of polyps develop throughout the colonic mucosa. Presentation in childhood has also been described; the earliest mutation-confirmed report was of a 4-year-old child. ${ }^{2,3}$ Adenomas can also be found in the small bowel; duodenal polyps can occur at as early as 5 years of age, ${ }^{2,4}$ whereas more distal polyps have been reported at 6 years of age. ${ }^{4}$ The association with extracolonic tumors, in particular with osteomas and soft tissue lesions, was first described by Gardner and Rich$a^{2} d s^{5}$ and has been named Gardner syndrome to emphasize this association in some families. Gardner fibromas (GAFs), which are associated with FAP, have only been pathologically defined more recently. ${ }^{5-8}$ These are superficial and poorly circumscribed tumor-like lesions that consist of formless sheets of thick collagen bundles with interspersed bland fibroblasts. ${ }^{6}$ Approximately $60 \%$ of GAFs are localized to the back and paraspinal regions, and they occur mostly in the first decade. ${ }^{6}$ It is noteworthy that when GAFs occur as part of FAP, they can precede the development of coIonic adenomas and, thus, may serve as sentinel lesions for the diagnosis of FAP in the proband or in apparently asymptomatic relatives. ${ }^{8}$ When sufficient clinical follow-up is available, previously reported cases of children presenting with GAF-associated FAP had a positive family history. ${ }^{6-11} \mathrm{Be}-$ cause up to one-third of FAP cases may be attributable to de novo mutations, ${ }^{12}$ one should be aware of the possibility that a child with a fibroma consistent with GAF may harbor FAP regardless of the family history. This concept is supported by the following case report, in which we describe the first (to our knowledge) molecularly proven case in which GAF was the sole, initial manifestation of FAP in the family. With GAFs being so frequently associated with FAP, it is of great importance that pathologists recognize this rare lesion and that subsequent appropriate investigations be conducted by pediatricians.

\section{CASE REPORT}

A 10-week-old girl without significant medical history presented with a right paraspinal mass at the level of $L 2$. The mass was noted initially by her parents at 3 weeks of age and demonstrated only modest enlargement over this interval. MRI of the spine revealed a mass that measured $20 \times 18 \times 4$ $\mathrm{mm}$ that was located deep to the subcutaneous fat and superficial to the paraspinal musculature. An excisional biopsy of the paraspinal tumor $(20 \times$ $18 \times 10 \mathrm{~mm}$ ) was performed and demonstrated histology suggestive of a neurofibroma. Immunohistochemistry was positive focally for the S-100 protein. At 5 months of age, a second paraspinal mass was noted on her left side at the level of T6-T7. This mass showed slow growth; its size was estimated to be $2 \times 4 \mathrm{~cm}$ at 14 months and $2 \times 5 \mathrm{~cm}$ at 2.5 years of age. This second mass was located just below the subcutaneous fat on MRI. The patient was followed in a multidisciplinary neurofibromatosis clinic, but clinical evaluation by dermatology and ophthalmology (fundoscopic examination), as well as a cranial MRI, failed to reveal any abnormalities at 11 months of age. This atypical presentation of neurofibromatosis prompted the excision of the second paraspinal mass $(45 \times 25 \times 15 \mathrm{~mm})$ at the age of 2.5 years. Histologic examination of this specimen revealed an infiltrating fibrogenic lesion composed of mature, dense hyaline collagen in which bland spindle cells of fibroblast type were present (Fig 1A). The process invaded the skeletal muscle and entrapped nerves. These features were consis- tent with a GAF. Retrospective review of the first excisional biopsy demonstrated similar features.

Given the known association between GAFs and FAP, the patient was referred for further investigation at 3 years of age. At the time of the assessment, history and the results of clinical examination were not otherwise supportive of FAP. The patient was the first child of a healthy nonconsanguineous French-Canadian couple. The patient's younger sister was reported to be healthy. Family history was negative for colorectal cancer and brain, hepatic, and skin tumors. The patient's paternal grandfather died of lymphoma at 71 years of age.

An abdominal ultrasound and serum $\alpha$-fetoprotein test were ordered, both of which yielded normal results. The child was considered too young to justify more invasive investigations, but while under follow-up, at 3 years 11 months, the patient experienced 2 brief episodes of bloody stools. Colonoscopy was then performed at 4 years 2 months of age and revealed 75 to 100 polyps from the rectum through to the cecum. Multiple adenomas were histopathologically confirmed. An upper endoscopy showed few discrete lesions in the duodenum, but biopsies revealed normal mucosa only. However, follow-up study at 4 years 7 months showed adenomatous changes without high-grade dysplasia. Six jejunal polyps $(<1 \mathrm{~cm})$ were also visualized by wireless-capsule endoscopy at 5 years 4 months. Analysis of the APC gene on peripheral blood leukocytes revealed a deleterious change at codon 1494 (c.4479_4480delGG p. Glu1494LysfsX19) that confirmed the clinical diagnosis of FAP. Both parents had normal colonoscopy results, and results of genetic testing for the daughter's mutation in blood leukocyte and buccal-swab DNA were nega- 
A

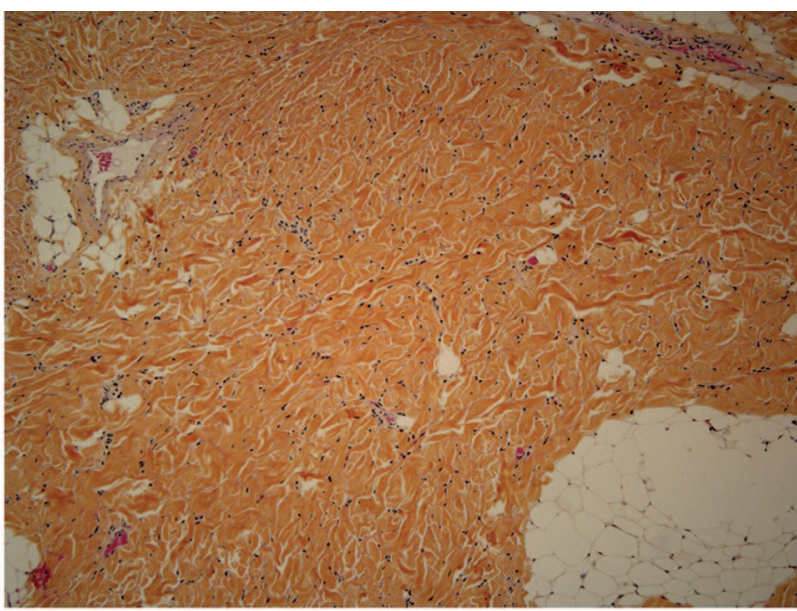

C

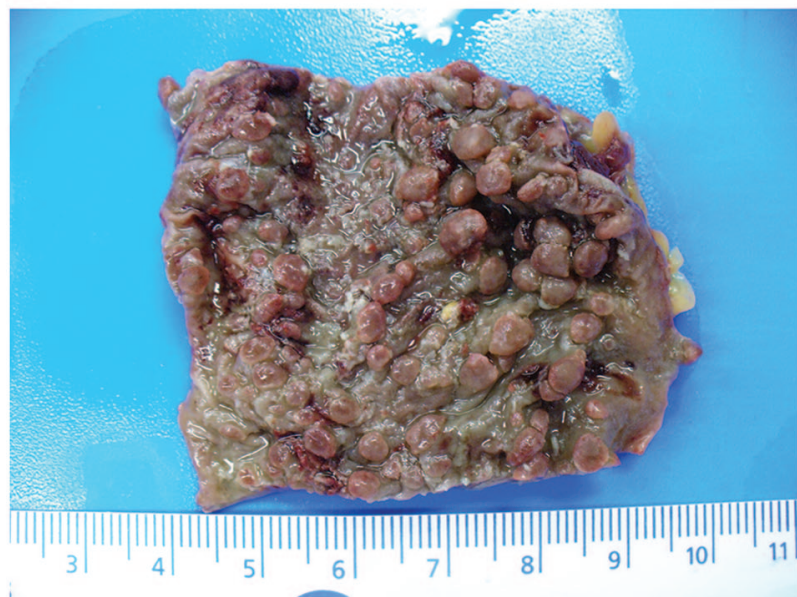

B

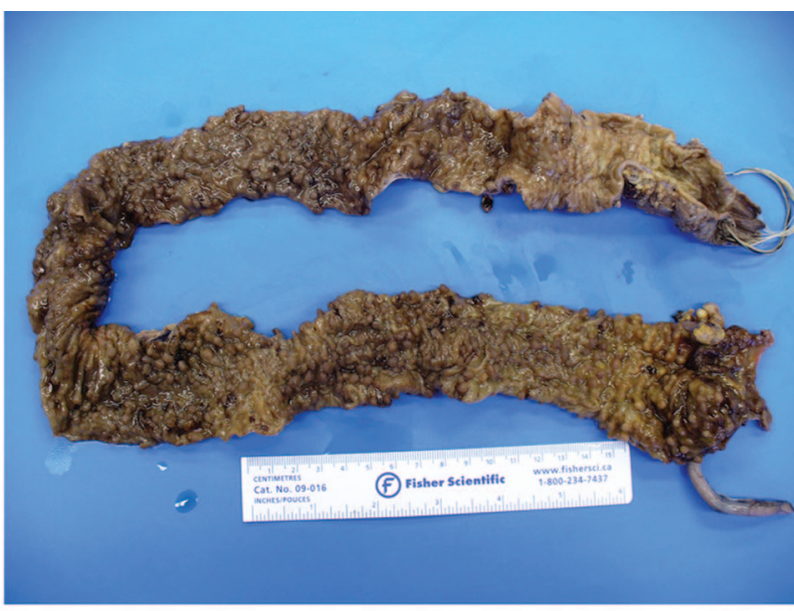

$\mathrm{D}$

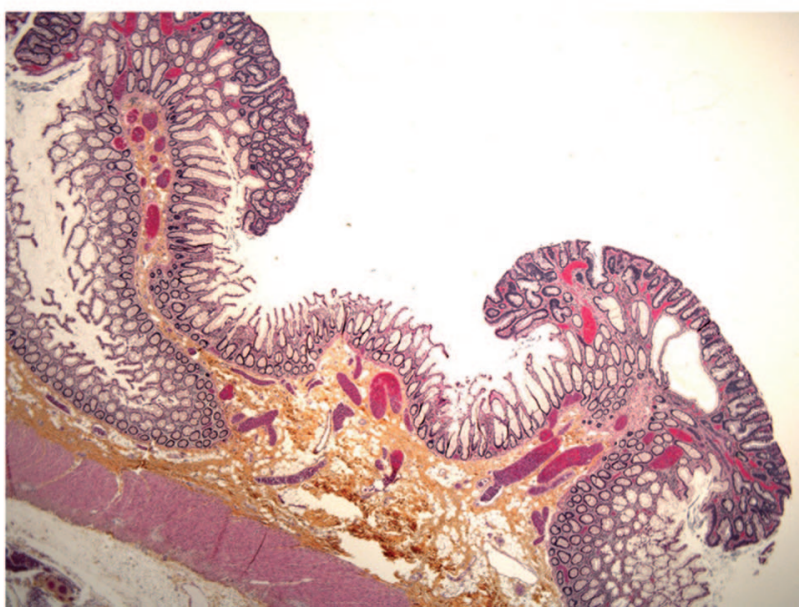

FIGURE 1

GAFs and severe polyposis in a 4-year-old child with FAP. A, Histologic view of the excised left paraspinal GAF showing mostly dense hyaline collagen with few fibroblast cells. B, Macroscopic view of the entire resected colon showing widespread $(>400)$ adenomas; a closer view is shown in C, and a histologic view of 2 adenomas is shown in $D$.

tive in each case. The younger sister also tested negative for the mutation.

A decision was made to perform a laparoscopic total proctocolectomy with ileoanal anastomosis at 4 years 7 months of age. Pathologic review of the resected colon did not show any malignant lesion. Approximately 400 polyps were visualized over the colonic mucosa (Fig 1 B-D)

\section{DISCUSSION}

Herein we report the first (to our knowledge) confirmed case of FAP with an APC mutation presenting in a child with an isolated GAF and an absence of family history of FAP or related disorders. Normal colonoscopy results and the absence of the $A P C$ mutation in both parents (leukocytes and buccal epithelial cells) suggest that it represents a de novo mutation, although germ-line mosaicism cannot be excluded. Coffin et al ${ }^{6}$ previously reported a series of 45 patients who presented with GAFs. Of those with sufficient information, 5 of 23 had no family history of FAP and none had a documented APC mutation or showed another manifestation of FAP, which led the authors to suggest that GAFs might be unrelated to FAP in a certain proportion of cases. However, because long-term clinical follow-up was lacking for these cases, this proportion may possibly be overestimated. Thus, there should be a high degree of suspicion of FAP when a mass with histology consistent with a GAF is identified.

The 2 excised lesions from our patient showed features consistent with GAFs, namely dense hyaline collagen with few spindle cells of fibroblast type and entrapment of nerves and skeletal muscle.6,8 The fact that the first lesion was confused initially with neurofibroma may be related, in part, to the presence of positive staining for S-100 protein by immunohistochemistry, which is related to the presence of nerves. Thus, the diagnosis of GAFs can be overlooked, especially in the absence of clinical or familial informa- 
tion that points to FAP. Given the importance of such information for cancer prevention, GAFs should be considered in the differential diagnosis when a hypocellular lesion with high collagen content presents in the pediatric context. GAFs cannot be distinguished histologically from nuchal-type fibromas (NTFs), but the predominance of GAFs in the first decade of life, compared with NTFs, should lead one to suspect GAFs in children. ${ }^{6}$ In addition, the implications are different, because NTFs are mainly associated with diabetes mellitus (44\%) and to a much lesser extent with FAP (6\%). ${ }^{10}$

Our case illustrates the importance of GAFs as sentinel lesions for FAP in the proband, as pointed out by Coffin et al. 6,8 The finding of this lesion should prompt an investigation in relatives for polyposis or extracolonic manifestations of FAP. The diagnosis should not be perceived to be unlikely even when there is a lack of supportive findings in the family, because $\sim 30 \%$ of APC mutations occur de novo..$^{12}$ Therefore, we recommend that FAP be excluded by genetic testing for APC mu-

\section{REFERENCES}

1. Galiatsatos P, Foulkes W. Familial adenomatous polyposis. Am J Gastroenterol. 2006; 101(2):385-398

2. Attard T, Tajouri T, Peterson K, Tinley S, Thorson A, Lynch H. Familial adenomatous polyposis in children younger than age ten years: a multidisciplinary clinic experience. Dis Colon Rectum. 2008;51(2):207-212

3. Caspari R, Friedl W, Mandl M, et al. Familial adenomatous polyposis: mutation at codon 1309 and early onset of colon cancer. Lancet. 1994;343(8898):629-632

4. Distante S, Nasioulas S, Somers G, et al. Familial adenomatous polyposis in a 5 year old child: a clinical, pathological, and molecular genetic study. J Med Genet. 1996; 33(2):157-160

5. Gardner E, Richards R. Multiple cutaneous and subcutaneous lesions occurring simultaneously with hereditary polyposis and osteomatosis. Am J Hum Genet. 1953;5(2):139-147 tations in any child with a fibroma consistent with GAF, even in the absence of any features of FAP. This testing should ideally predate colonoscopic evaluation, which generally commences at 10 to 12 years of age in familial cases of FAP, especially because colonoscopy for younger children is an invasive procedure with potential risks. ${ }^{13}$ In that respect, the early onset and severity of both the colonic polyposis and the duodenal adenomas of our case are quite unusual. Indeed, to our knowledge, this is the earliest case of mutation-confirmed FAP reported in the literature. At the present time, there is no evidence that polyposis manifests earlier in patients with GAFs; thus, no specific recommendation regarding the timing of an endoscopy can be made on the basis of identifying a GAF.

The mutation observed in the present case was reported before in 3 apparently unrelated individuals (www.umd. be/APC/). However, clinical details of these cases are not available. Correlation between the site of the mutation with the occurrence of GAFs is not

6. Coffin C, Hornick J, Zhou H, Fletcher C. Gardner fibroma: a clinicopathologic and immunohistochemical analysis of 45 patients with 57 fibromas. Am J Surg Pathol. 2007; 31(3): $410-416$

7. Michal M, Boudova L, Mukensnabl P. Gardner's syndrome associated fibromas. Pathol Int. 2004;54(7):523-526

8. Wehrli B, Weiss S, Yandow S, Coffin C. Gardner-associated fibromas (GAF) in young patients: a distinct fibrous lesion that identifies unsuspected Gardner syndrome and risk for fibromatosis. Am J Surg Pathol. 2001;25(5):645-651

9. Diwan A, Graves E, King J, Horenstein M. Nuchal-type fibroma in two related patients with Gardner's syndrome. Am J Surg Pathol. 2000;24(11):1563-1567

10. Michal M, Fetsch J, Hes 0 , Miettinen M. Nuchal-type fibroma: a clinicopathologic known, because there are few patients for whom the mutation has been described, and extensive follow-up is not available in most cases. It is notable that 1 case of GAF with an unspecified mutation in exon 15 has been reported, as seen in our patient. ${ }^{8}$ Finally, the association between the specific mutation of our patient and her particularly severe and unusual phenotype is unknown. Profuse polyposis is usually associated with mutations between codons 1250 and 1464, ${ }^{14}$ and particularly early onset of aggressive disease in multiple individuals with mutation in codon 1309 has been reported. 2,3

\section{CONCLUSIONS}

To our knowledge, this is the first report of neonatal GAFs as the presenting feature of a sporadic case of FAP with an unusually severe and early onset. It is also the earliest report of coIonic and small bowel involvement in FAP. It illustrates the need to exclude FAP in a child who harbors this type of superficial lesion, even in the absence of any familial evidence of the disease.

study of 52 cases. Cancer. 1999;85(1): 156-163

11. Michal M. Non-nuchal-type fibroma associated with Gardner's syndrome: a hithertounreported mesenchymal tumor different from fibromatosis and nuchal-type fibroma. Pathol Res Pract. 2000;196(12):857-860

12. Rustin $R$, Jagelman D, McGannon E, Fazio V, Lavery I, Weakley F. Spontaneous mutation in familial adenomatous polyposis. Dis CoIon Rectum. 1990;33(1):52-55

13. Giardiello F, Brensinger J, Petersen G. AGA technical review on hereditary colorectal cancer and genetic testing. Gastroenterology. 2001;121(1):198-213

14. Nagase H, Miyoshi Y, Horii A, et al. Correlation between the location of germ-line mutations in the $A P C$ gene and the number of colorectal polyps in familial adenomatous polyposis patients. Cancer Res. 1992;52 (14):4055-4057 


\section{Neonatal Gardner Fibroma: A Sentinel Presentation of Severe Familial Adenomatous Polyposis}

Sebastien Levesque, Najma Ahmed, Van-Hung Nguyen, Ayoub Nahal, Miriam

Blumenkrantz, Pramod Puligandla, George Chong and William D. Foulkes

Pediatrics originally published online November 8, 2010;

\section{Updated Information \&} Services

Permissions \& Licensing including high resolution figures, can be found at:

http://pediatrics.aappublications.org/content/early/2010/11/08/peds.2 010-1045

Information about reproducing this article in parts (figures, tables) or in its entirety can be found online at:

http://www.aappublications.org/site/misc/Permissions.xhtml

Reprints
Information about ordering reprints can be found online: http://www.aappublications.org/site/misc/reprints.xhtml

\section{American Academy of Pediatrics}




\section{PED I ATR I C S}

OFFICIAL JOURNAL OF THE AMERICAN ACADEMY OF PEDIATRICS

\section{Neonatal Gardner Fibroma: A Sentinel Presentation of Severe Familial Adenomatous Polyposis}

Sebastien Levesque, Najma Ahmed, Van-Hung Nguyen, Ayoub Nahal, Miriam Blumenkrantz, Pramod Puligandla, George Chong and William D. Foulkes Pediatrics originally published online November 8, 2010;

The online version of this article, along with updated information and services, is located on the World Wide Web at:

http://pediatrics.aappublications.org/content/early/2010/11/08/peds.2010-1045

Pediatrics is the official journal of the American Academy of Pediatrics. A monthly publication, it has been published continuously since 1948. Pediatrics is owned, published, and trademarked by the American Academy of Pediatrics, 345 Park Avenue, Itasca, Illinois, 60143. Copyright (C) 2010 by the American Academy of Pediatrics. All rights reserved. Print ISSN: 1073-0397.

\section{American Academy of Pediatrics}

